\title{
Colliding Beams
}

\section{from a High Energy Physics Correspondent}

AN experiment which yields in all qualitative respects the theoretically predicted results and which has intrinsic novelty and elegance as well was reported at the recent High Energy Physics Conference in Heidelberg. H. Joos of the DESY group in West Germany reported the results of an experiment performed by a Russian group at Novosibirsk which has studied the annihilation of an electron and a positron and the subsequent production of a pair of $\pi$ mesons with opposite charges. This experiment, like similar experiments being performed at Orsay in France and Stanford in the United States, relies on the very recent experimental technique of noaking two beams of particles collide. The advantage of this method lies in the relativistic kinematics of the situation in which the square of the beam energy in the colliding beam is a true measure of the usable energy. In the more conventional situations, with a beam striking a stationary target, usable energy is proportional to the product of beam energy and the mass of the target particle.

In principle, it is quite simple to perform a colliding beam experiment such as that carried out at DESY. The first step is to build a storage ring, which consists of the field magnets and vacuum system for a small synchrotron. This device can then be fed with electrons from some conventional synchrotron or linear accelerator until a considerable current accumulates. A similar beam of positrons is then built up, either as a separate beam in the same storage ring (but travelling in the opposite direction because of the charge) or in another storage ring so arranged as to form a figure of eight with the first. The two beams can then be made to collide and conventional detection methods used to observe the reaction products. Although the relative probability of a collision between the beams is low compared with that when a solid or liquid target is used, this disadvantage is somewhat offset by the several chances of collision afforded by the repeated intersections of the orbiting particles. In practice, the difficulties, arising chiefly from resonance effects which cause beam instabilities, are enormous and have taken several years to understand, so that the new experimentthe first in which a strongly interacting final state has been studied-represents a landmark in the field and is a tribute to the skill of the experimentalists involved.

Several years ago, studies of nuclear form factors obtained from measurements on the scattering of electrons by nucleons suggested the existence of a neutral particle with unit spin and a mass of several hundred. $\mathrm{MeV}$. This was later confirmed by the discovery of the $\rho$ meson at a mass of some $760 \mathrm{MeV}$ in pion production experiments. By invoking what is believed to be one of the basic principles of physics known as crossing symmetry, it is possible to infer that if this $p$ meson couples electromagnetically to the electrons, which would account for the form factors, then an electron-positron. pair should annihilate electromagnetically to form such a $p$ meson which would then decay, by strong interaction, into a pair of charged pions. The new experiment confirms this concept in a most striking manner. When the number of pion pairs is plotted against energy, an almost classic Breit-Wigner resonance decay curve is seen with a peak at a mass value of $764 \mathrm{MeV}$ and a width of $93 \pm 15 \mathrm{MeV}$.
Although the immediate interest lies in the novelty of the experimental technique, detailed experiments along these lines seem certain to suggest a major revision of current ideas.

\section{Reliable Electricity}

\section{from a Correspondent}

CAN the economic consequences of unreliability of electricity supply be properly quantified? From whose viewpoint should this task be attempted? What is the place of statistical and probability methods in such studies? How can the experience of various countries in this field be collated and compared? These and many other related questions were discussed at a conference last week organized by the Power Division of the Institution of Electrical Engineers. About 250 delegates, including 40 from 14 overseas countries, participated in three full-day sessions devoted to the problems of generation, transmission and distribution connected with this issue. It must be said at once that the questions remained largely unanswered, although many preliminary suggestions were advanced.

It is rare, if not unprecedented, for the product of the electricity supply industry to be examined in this way. In both national and international forums, the manufacturers of electrical plant are accustomed to describe the design and performance of their products and to face questions and criticism from customers and competitors. Publications by electricity supply engineers are commonly restricted to planning and operational problems. The papers at this conference clearly showed the reluctance felt by some authors to unveil the basis for the cost-benefit calculations which they may have attempted. Unfortunately their major customers, the large electricity using industries, played only a small part in the conference and, despite some shrewd blows, were heavily outnumbered by the supply side. The plant manufacturers appeared uncertain as to their proper role in these unusual proceedings and remained rather quiet.

The conference might therefore have been a rather tame affair, but it was saved by the excellent, frank and good-humoured contributions from abroad. A Danish engineer drew some interesting contrasts between electronic and nuclear equipment design and that of power plant and systems. Might it perhaps be both cheaper and more reliable to employ a certain amount of duplication and redundancy while lowering the performance specification for individual items?

The same speaker added, quixotically, that he felt a power cut now and then might serve to remind us of our ever increasing dependence upon the machinery of civilization and progress. He was not supported in this by a Swedish colleague, who was sure that their job was to build the biggest possible market for electricity by offering consumers what they want to buy. (This speaker also made the possibly significant point that the Swedish State Power Board supplies only 40 per cent of the country's electricity. Comparisons with other undertakings thus remain possible and provide a valuable stimulus to efficiency.) An engineer from Electricité de France was the contributor who laid most stress upon the concept of "loss to the community" resulting from failures of supply, and who demonstrated how far l'esprit cartésien pene- 